\title{
Generalized lichen amyloidosis
}

\section{Safae Maouni', Lina Mezni', Asmae Abdelmoutalib', Kaoutar Znati², Mariame Meziane', Nadia Ismaili' ${ }^{1}$, Karima Senouci ${ }^{1}$, Laila Benzekri ${ }^{1}$}

${ }^{1}$ Department of Dermatology-Venereology, Ibn Sina University Hospital, Mohammed V University, Rabat, Morocco, ${ }^{2}$ Department of Anatomopathology, Ibn Sina University Hospital, Mohammed V University, Rabat, Morocco

Corresponding author: Dr. Safae Maouni, E-mail: maouni.safae88@gmail.com

Sir,

Lichen amyloidosis (LA) is a primary cutaneous amyloidosis characterized clinically by papular lesions with a usual localization on the legs. The generalized form is uncommon. We report a new atypical case of lichen amyloidosis characterized by generalized lesions and successfully treated with a new therapeutic combination: acitretin and trichloroacetic acid at 50\%.

A 57-year-old female with a history of high blood pressure under treatment consulted for very itchy papular lesions evolving for the last six years and treated with emollients, dermocorticoids, and antihistamines but without improvement. The lesions were initially located on the legs but then gradually spread to the rest of the body. A dermatological examination found papular, flesh-colored lesions $2-3 \mathrm{~mm}$ in diameter, some of them keratotic, rough on palpation, resting on hyperpigmented plates, associated with scratching lesions, and located on the arms, forearms, thighs, legs, back, and neck (Figs. 1 and 2). A skin biopsy revealed an acanthotic and papillomatous epidermis and orthokeratotic hyperkeratosis with amorphous and cracked eosinophilic deposits in the papillary dermis (Fig. 3) easily visible with standard staining and birefringent with the Congo red stain. The rest of the biological and morphological assessments revealed no association with endocrine neoplasia. The diagnosis of generalized lichen amyloidosis was established. The patient was treated with acitretin 25 mg daily in combination with trichloroacetic acid at $50 \%$ (one application every fifteen days). There was a decrease in the pruritus from the first month and a good evolution of the cutaneous lesions after one year of follow-up.



Figure 1: (a-b) Generalized lichen amyloidosis: multiple papules on hyperpigmented plaques on the upper and lower limbs.

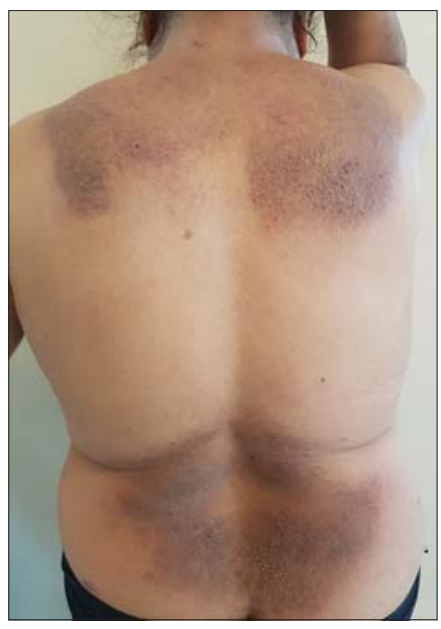

Figure 2: Generalized lichen amyloidosis: multiple papules on hyperpigmented plaques on the back.

The generalized form of lichen amyloidosis is rare and often associated with other dermatoses that aggravate the pruritus, such as lichen planus [1], chronic urticarial [2], atopic dermatitis, prurigo, and mycosis fungoides. In our patient, data from the interview, a clinical examination, and additional examinations could not reveal an associated aggravating factor.

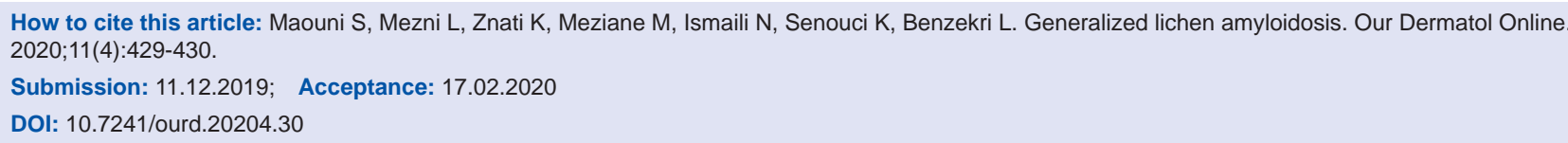




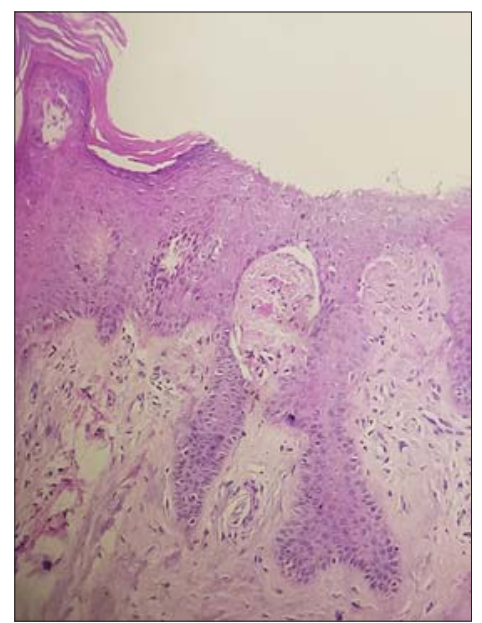

Figure 3: Generalized lichen amyloidosis and histologic findings: an acanthotic and papillomatous epidermis with orthokeratotic hyperkeratosis; globular eosinophilic deposits throughout the papillary dermis (H\&E, 100x).

Two theories have been proposed to explain the mechanism of cutaneous amyloidosis: the apoptotic theory, which involves the transformation of keratin from apoptotic keratinocytes into amyloid bodies, and another theory, which proposes that the cutaneous amyloid deposits result from direct secretion by keratinocytes [3]. The therapeutic management of this clinical form is very delicate and several therapeutic methods have been proposed by the literature. Oral retinoids were prescribed in some cases, but the clinical course was variable depending on the genetic terrain and the extent of the disease. Indeed, acitretin works through its anti-inflammatory and anti-proliferative action by promoting keratinocyte differentiation and through its action on apolipoprotein $\mathrm{E}$, which allows a reduction in amyloid deposits $[4,5]$.
Given the synergistic effects of the two treatments and the good clinical response observed in our patient, this therapeutic combination may be recommended in the treatment of cases of generalized LA resistant to usual treatments.

\section{Consent}

The examination of the patient was conducted according to the principles of the Declaration of Helsinki.

The authors certify that they have obtained all appropriate patient consent forms, in which the patients gave their consent for images and other clinical information to be included in the journal. The patients understand that their names and initials will not be published and due effort will be made to conceal their identity, but that anonymity cannot be guaranteed.

\section{REFERENCES}

1. Matsumura N, Yamamoto T. Lichen amyloidosis associated with chronic renal failure. Our Dermatol Online. 2017;8:237-8.

2. Smogorzewski JM, Rodriguez L, Young L. Symmetric lichen amyloidosis: an atypical location on the bilateral extensor surfaces of the arms. Cutis. 2019;103:E9-10.

3. Katayama C, Hayashida Y, Sugiyama S, Shiohara T, Aoyama Y. Postherpetic hypohidrosis-related isotopic response associated with lichen planus and lichen amyloidosis. Eur J Dermatol. 2019;29:233-4.

4. Robinson CG, Jahan-Tigh RR. Successful treatment of lichen amyloidosis with trichloroacetic acid peels. Dermatol Surg. 2019;45:854-7.

5. Weidner T, Illing T, Elsner P. Primary localized cutaneous amyloidosis: a systematic treatment review. Am J Clin Dermatol. 2017;18:629-42.

Copyright by Safae Maouni, et al. This is an open access article distributed under the terms of the Creative Commons Attribution License, which permits unrestricted use, distribution, and reproduction in any medium, provided the original author and source are credited.

Source of Support: Nil, Conflict of Interest: None declared. 